\title{
RESEARCH
}

Open Access

\section{CXCR5-negative natural killer cells ameliorate experimental autoimmune myasthenia gravis by suppressing follicular helper T cells}

Chun-Lin Yang ${ }^{1}$, Peng Zhang ${ }^{2}$, Ru-Tao Liu², Na Zhang ${ }^{2}$, Min Zhang ${ }^{2}$, Heng Li ${ }^{2}$, Tong Du' ${ }^{1}$, Xiao-Li Li², Ying-Chun Dou ${ }^{3}$ and Rui-Sheng Duan ${ }^{1,2^{*}}$ (D)

\begin{abstract}
Background: Recent studies have demonstrated that natural killer (NK) cells can modulate other immune components and are involved in the development or progression of several autoimmune diseases. However, the roles and mechanisms of NK cells in regulating experimental autoimmune myasthenia gravis (EAMG) remained to be illustrated.

Methods: To address the function of NK cells in experimental autoimmune myasthenia gravis in vivo, EAMG rats were adoptively transferred with splenic NK cells. The serum antibodies, and splenic follicular helper $T$ (Tfh) cells and germinal center B cells were determined by ELISA and flow cytometry. The roles of NK cells in regulating Tfh cells were further verified in vitro by co-culturing splenocytes or isolated T cells with NK cells. Moreover, the phenotype, localization, and function differences between different NK cell subtypes were determined by flow cytometry, immunofluorescence, and ex vivo co-culturation.
\end{abstract}

Results: In this study, we found that adoptive transfer of NK cells ameliorated EAMG symptoms by suppressing Tfh cells and germinal center B cells. Ex vivo studies indicated NK cells inhibited CD4 ${ }^{+} T$ cells and Th cells by inducing the apoptosis of T cells. More importantly, NK cells could be divided into CXCR5 ${ }^{-}$and $\mathrm{CXCR5}^{+}$NK subtypes according to the expression of CXCR5 molecular. Compared with CXCR5 NK cells, which were mainly localized outside B cell zone, CXCR5 ${ }^{+}$NK were concentrated in the B cell zone and exhibited higher expression levels of IL-17 and ICOS, and lower expression level of CD27. Ex vivo studies indicated it was CXCR5 ${ }^{-}$NK cells not CXCR5 ${ }^{+} \mathrm{NK}$ cells that suppressed CD4 ${ }^{+} T$ cells and Tfh cells. Further analysis revealed that, compared with $\mathrm{CXCR5}^{-} \mathrm{NK}$ cells, CXCR5 ${ }^{+}$ NK cells enhanced the ICOS expression of Tfh cells.

Conclusions: These findings highlight the different roles of CXCR5 ${ }^{-} \mathrm{NK}$ cells and $\mathrm{CXCR5^{+ }}$ NK cells. It was $\mathrm{CXCR5}{ }^{-} \mathrm{NK}$ cells but not $\mathrm{CXCR5^{+ }}$ NK cells that suppressed Tfh cells and inhibited the autoimmune response in EAMG models.

Keywords: Experimental autoimmune myasthenia gravis, Natural killer cells, C-X-C chemokine receptor 5 (CXCR5), Follicular helper T cells

\footnotetext{
* Correspondence: ruisheng_duan@yahoo.com

${ }^{1}$ Department of Neurology, Shandong Provincial Qianfoshan Hospital,

Shandong University, No. 16766, Jingshi Road, Jinan 250014, People's

Republic of China

${ }^{2}$ Department of Neurology, The First Affiliated Hospital of Shandong First

Medical University, Jinan 250014, People's Republic of China

Full list of author information is available at the end of the article
}

(c) The Author(s). 2019 Open Access This article is distributed under the terms of the Creative Commons Attribution 4.0 International License (http://creativecommons.org/licenses/by/4.0/), which permits unrestricted use, distribution, and reproduction in any medium, provided you give appropriate credit to the original author(s) and the source, provide a link to the Creative Commons license, and indicate if changes were made. The Creative Commons Public Domain Dedication waiver (http://creativecommons.org/publicdomain/zero/1.0/) applies to the data made available in this article, unless otherwise stated. 


\section{Introduction}

Myasthenia gravis (MG) is an antibody-mediated autoimmune disease which is mainly caused by antibodies targeting acetylcholine receptor (AChR) at the postsynaptic neuromuscular junction membrane, leading to impaired signal transduction, muscle weakness, and fatigability [1]. Other MG-specific autoantibodies include antibodies against muscle-specific kinase and lipoprotein receptorrelated protein 4 [2].

Follicular helper $\mathrm{T}$ (Tfh) cells are the major $\mathrm{CD}^{+} \mathrm{T}$ cell subset involved in humoral immune response. Tfh cells, which are characterized by the expression of CXCR5, can migrate into B cell follicles, and promote the proliferation, differentiation, and antibody classswitching of B cells. Tfh cells express high levels of inducible co-stimulator (ICOS), programmed death 1 (PD-1), and IL-21, all of which are essential for antibody production and generation memory $B$ cells with high-affinity antibodies. Exaggerated Tfh cell responses have been demonstrated in a broad range of autoimmune diseases such as systemic lupus erythematosus and autoimmune thyroid diseases [3]. Altered circulating Tfh cells or Tfh cell subtypes in patients with myasthenia gravis have also been reported in several recent literatures $[4,5]$. It was also demonstrated that intra-thymic Tfh cells could interact with B cells, leading to ectopic germinal centers formation and anti-AChR antibody production [6]. Reduction of Tfh cells could ameliorate the symptom of experimental autoimmune myasthenia gravis (EAMG) in mice [7]. All of these studies suggest a pathogenic role of Tfh in MG/EAMG.

Natural killer (NK) cells are large granular lymphocytes with the capacities to directly recognize and kill foreign, infected, and malignant cells [8]. Growing evidences from mouse and human studies demonstrate that NK cells can also modulate other aspects of the immune system and are involved in the development or progression of the autoimmune diseases, through their production of numerous cytokines and chemokines or by perforin-mediated cell death [8-10].

NK cells limited autoimmune occurrence by controlling $\mathrm{CD}^{+} \mathrm{T}$ cell and $\mathrm{CD}^{+} \mathrm{T}$ cell responses in the TNF-related apoptosis-inducing ligand (TRAIL) or perforin-dependent manner [11-13]. It has been shown that NK cells isolated from patients with MS had decreased frequency and effector function [10]. Transfer of acetylcholine-producing NK cells into the cerebral ventricles of experimental autoimmune encephalomyelitis (EAE) mice attenuated brain and spinal cord damage via modulation of infiltrating monocytes and macrophages [14]. Recent studies also demonstrated an inhibitory role of NK cells in regulation of Tfh cells in lymphocytic choriomeningitis virus model and NPKLH (4-hydroxy-3-nitrophenylacetyl hapten conjugated to Keyhole Limpet Hemocyanin) immunization model $[15,16]$. However, it is unclear whether NK cells could modulate Tfh cells and their subtypes in EAMG model and other autoimmune diseases.

\section{Materials and methods \\ Animals}

Female Lewis rats (6-8 weeks) were purchased from Vital River Laboratories (Beijing, China), kept under specific pathogen-free conditions and 12/12 light-dark schedule, and provided with standard pellet diet and water ad libitum. Animals were killed by isoflurane inhalation and decapitation. All the experimental protocols were approved by the institutional ethics committee.

\section{Induction of EAMG}

Rat AChR97-116 peptide (DGDFAIVKFTKVLLDYT$\mathrm{GHI}$, corresponding to rat AChR alpha subunit, were purchased from China Peptides Co. Ltd (Shanghai, China). Rat EAMG models were induced as previously reported [17]. In brief, the rats were immunized subcutaneously at the tail base with a total $200 \mu \mathrm{l}$ emulsion containing $75 \mu \mathrm{g}$ of rat AChR97-116 peptide in phosphate buffer saline (PBS) emulsified in an equal volume of complete Freund's adjuvant containing $1 \mathrm{mg}$ mycobacterium tuberculosis (strain H37RA; Difco, Detroit, MI, USA). Then, the rats were boosted on day 30 with the same dose of AChR97-116 in incomplete Freund's adjuvant. After the first immunization, body weights and clinical scores were assessed every other day in the blinded manner by two researchers at the same time as follows: 0, normal strength and no abnormalities; 1, mildly decreased activity and weak grip or cry, more evident at the end of exercise; 2, clinical signs present before exercise (tremor, head down, hunched posture, weak grip); 3, severe clinical signs present before exercise, no grip, moribund; and 4, dead.

\section{Induction of experimental autoimmune neuritis (EAN)}

Bovine peripheral myelin (BPM) was isolated from fresh adult bovine cauda equina as previously reported [18]. To induce EAN rat model, ten female Lewis rats, aged 6-8 weeks, were subcutaneously immunized with $200 \mu \mathrm{l}$ emulsion containing $1 \mathrm{mg}$ BPM in saline and an equal volume of complete Freund's adjuvant containing $0.3 \mathrm{mg}$ Mycobacterium tuberculosis (strain H37RA; Difco, Detroit, MI, USA) at the tail base. At the day 13 postimmunization (p.i.), the rats were sacrificed and the spleens were obtained for flow cytometry analysis.

\section{Flow cytometry and intracellular cytokine staining}

Spleens or lymph nodes were minced through the 70$\mu \mathrm{m}$ cell strainers to generate single-cell suspensions. The red blood cells of splenic single-cell suspensions were 
lysed by RBC lysis buffer (Biolegend) for 5 min on ice. The cells were stained according to standard protocols with the following antibodies: anti-rat CD25 (OX39; Invitrogen), CD161 (10/78; BD Pharmingen), CD80 (3H5; eBioscience), CD86 (24F; Biolegend), CD4 (OX35; eBioscience), MHC-II (OX-6; Biolegend), Foxp3 (FJK16S; eBioscience), IL-17A (eBio17B7; eBioscience), CD3 (1F4; Biolegend), CD103 (OX62; Biolegend), IFN- $\gamma$ (DB1; Invitrogen), ICOS (C398.4A; Biolegend), B220 (HIS24; eBioscience), CXCR5 (EPR8837; Abcam), and peanut agglutinin (PNA) (Vector Laboratories). Unconjugated primary antibodies were detected with Alexa Fluor 488-conjugated anti-rabbit IgG (Abcam). The following monoclonal antibodies were used for mouse cell staining: CD4 (GK1.5; eBioscience), NK1.1 (PK136; Biolegend), PD-1 (29F.1A12; Biolegend), CD3 (145-2C11; eBioscience), CXCR5 (2G8; BD Pharmingen), CXCR5 (L138D7; Biolegend), and CD19 (eBio1D3; eBioscience).

For intracellular cytokine staining, cells were stimulated for $5 \mathrm{~h}$ at $37^{\circ} \mathrm{C}$ with cell stimulation cocktail (eBioscience). Stimulated cells were then pre-incubated with PBS containing $0.5 \%$ bovine serum albumin and stained for $30 \mathrm{~min}$ at $4{ }^{\circ} \mathrm{C}$ with various combinations of fluorescently tagged antibodies. After washing, cells were fixed in $2 \%$ paraformaldehyde and then permeabilized using intracellular fixation and permeabilization buffer set (eBioscience, 88-8824-00) and then stained with antibodies specific for various cytokines. Flow cytometry was performed on BD FACS Aria II (BD Biosciences).

\section{Isolation of NK cells by magnetic beads and FACS}

The isolation of NK cells by magnetic cell sorting was performed in two steps according to the manufacturer's instructions. Briefly, splenocytes were stained with Alexa Fluor 647-conjugated anti-rat CD3 (Biolegend) $\mathrm{mAb}$ and PE-conjugated anti-rat CD161 (BD Biosciences) mAb. Then, the cells were incubated with anti-Alexa Fluor 647 microbeads (Miltenyi Biotec). Subsequently, the cell suspension was loaded on a selection column placed in a magnetic field to deplete the CD3-positive cells. Secondly, the flow-through fraction was incubated with anti-PE microbeads (Miltenyi Biotec). Finally, the cell suspension was loaded on a selection column placed in a magnetic field and the magnetically retained fraction was the NK cell fraction. The purity of NK cells, as analyzed by flow cytometry, was routinely $>90 \%$.

Isolation of NK cells for ex vivo culture was performed by sorting $\mathrm{CD}^{-} \mathrm{CD} 161^{\text {bright }}$ or $\mathrm{CD} 3^{-} \mathrm{NK} 1.1^{+}$cells from rat or mouse spleen using a BD FACS Aria II. The purities were about $95 \%$. To isolate $\mathrm{CXCR5}^{+}$and CXCR5 NK cells, the NK1.1- or CD161-positive cells were enriched from mouse or rat splenocytes by magnetic cell sorting. Then, $\mathrm{CXCR}^{+}$and $\mathrm{CXCR}^{-} \mathrm{NK}$ cells were sorted from NK1.1 or CD161 positive fraction by BD
FACS Aria II with the purity about $85 \%$. Isolation of $\mathrm{T}$ cells for ex vivo culture was performed by sorting $\mathrm{CD}^{+}$ $\mathrm{T}$ cells from rat or mouse spleen using a BD FACS Aria II with the purity about $95 \%$.

\section{NK cell adoptive transfer}

$5 \times 10^{6}$ magnetically sorted NK cells in $300 \mu$ l PBS were injected intravenously (i.v.) into recipient EAMG rats twice at the day before the first and second immunization, respectively. The control rats received the same volumes of PBS at the corresponding time.

To test the migration of NK cells in vivo, magnetically sorted NK cells or BD FACS Aria II sorted CXCR $5^{+}$and CXCR5 ${ }^{-} \mathrm{NK}$ cells were labeled for $15 \mathrm{~min}$ at $37^{\circ} \mathrm{C}$ with the $2 \mu \mathrm{M}$ fluorescent dye carboxyfluorescein diacetate, succinimidyl ester (CFSE) (CFDA-SE, Molecular Probes), washed, and transferred i.v. $\left(5 \times 10^{6}\right.$ cells for NK cells or $0.5 \times 10^{6}$ cells for $\mathrm{CXCR}^{+}$and CXCR5 ${ }^{-} \mathrm{NK}$ cells) to recipient immunized rats. Two days later, the spleens were harvested and applied for immunofluorescent stain.

\section{ELISA testing for antibody levels and affinities}

Rat serum anti-AChR97-116 IgG, IgG1, IgG2a, and IgG2b antibody levels were detected by ELISA as described previously [17]. The following reagents were used: biotin rabbit anti-rat IgG (Poly4054; BioLegend), IgG1 (MRG1-58; BioLegend), IgG2a (MRG2a-83; BioLegend), IgG2b (MRG2b-85; BioLegend), streptavidinhorseradish peroxidase (Biosynthesis Biotechnology), and tetramethylbenzidine (TMB) substrate (Tiangen Biotechnology, Beijing, China). The serum anti-AChR97116 IgG antibody affinities were determined by the thiocyanate method as described previously [19]. OD values were read at a wavelength of $450 \mathrm{~nm}$ by using a microplate ELISA reader.

\section{Immunofluorescence microscopy}

Cryosections of $8 \mu \mathrm{m}$ were fixed in cold acetone and stained with mouse anti-rat CD161 (10/78; BD Pharmingen) and fluorescein-conjugated PNA (Vector Laboratories) or rabbit anti-rat CXCR5 (Abcam), followed by PEconjugated rabbit anti-mouse IgG or Alexa Fluor 488conjugated anti-rabbit IgG (Abcam). For NK cell tracing experiment, cryosections were fixed and stained with Dylight 594-conjugated anti-rat IgM (Abcam). Images were captured with fluorescence microscopy (Olympus FSX100, Tokyo, Japan).

\section{Cell culture}

Immunized rat or mouse splenocytes were re-suspended to $1-2 \times 10^{6} / \mathrm{ml}$ in RPMI 1640 (containing $2.05 \mathrm{mM}$ glutamine; HyClone, Beijing, China) supplemented with $1 \%(\mathrm{v} / \mathrm{v})$ penicillin-streptomycin (containing 10,000 IU/ml penicillin and $10,000 \mu \mathrm{g} / \mathrm{ml}$ streptomycin; HyClone, Logan, UT, 
USA) and 10\% (v/v) fetal bovine serum (FBS; Biological Industries, Israel) for the following experiments. (i) Rat splenocytes were co-cultured with NK cells or not (splenocytes: $\mathrm{NK}=5: 1$ ) in the presence of IL-2 for three days. (ii) Mouse splenocytes or T cells were co-cultured with NK cells or not (splenocytes: $\mathrm{NK}=5: 1$ ) in the presence of $1 \mu \mathrm{g} / \mathrm{ml}$ anti-CD3, $1 \mu \mathrm{g} / \mathrm{ml}$ anti-CD28, and $10 \mathrm{ng} / \mathrm{ml} \mathrm{IL-15}$ for 3 days. (iii) Splenocytes or $\mathrm{T}$ cells were co-cultured with CXCR5 ${ }^{-} \mathrm{NK}$ cells, $\mathrm{CXCR} 5^{+} \mathrm{NK}$ cells, or not (splenocytes or $\mathrm{T}: \mathrm{NK}=5: 1$ ) in the presence of anti-CD3, anti-CD28, and IL-15 for 3 days. (iv) Mouse splenocytes were cultured with IL-15 or not in the presence of anti-CD3 and anti-CD28 for 3 days. (v) Mouse splenocytes were labeled with CFSE and cultured with IL-15 or not in the presence of lipopolysaccharide (LPS) for three days. Then, the cells were harvested, stained with corresponding antibodies and detected by using a flow cytometry.

\section{Statistical analysis}

Results are routinely displayed as mean \pm SEM, with statistical differences between experimental groups determined using a two-tailed unpaired Student's $t$ test, one-way ANOVA, and Spearman correlation test, where a $p$ value of $<0.05$ was deemed significant. Graphs were produced, and statistical analyses were performed using GraphPad Prism.

\section{Results}

NK cells ameliorate EAMG symptoms and reduce serum anti-AChR97-116 antibody levels and antibody affinities To test for the regulatory roles of NK cells in EAMG, splenic NK cells $\left(5 \times 10^{6}\right)$ from donor rats were isolated and transferred into recipient EAMG rats twice at the day before the first and second immunization, respectively. Compared with control rats, NK cell-treated rats had lower clinical scores (Fig. 1b), associated with reductions of anti-AChR97-116 IgG2a antibody levels (Fig. 1c). There was a trending but not statistically significant decrease of anti-AChR97-116 IgG antibody affinities in NK cell-treated group ( $p=0.09$, Fig. 1d). However, we did not find any differences in the concentrations of antiAChR97-116 IgG, IgG1, or IgG2b between those two
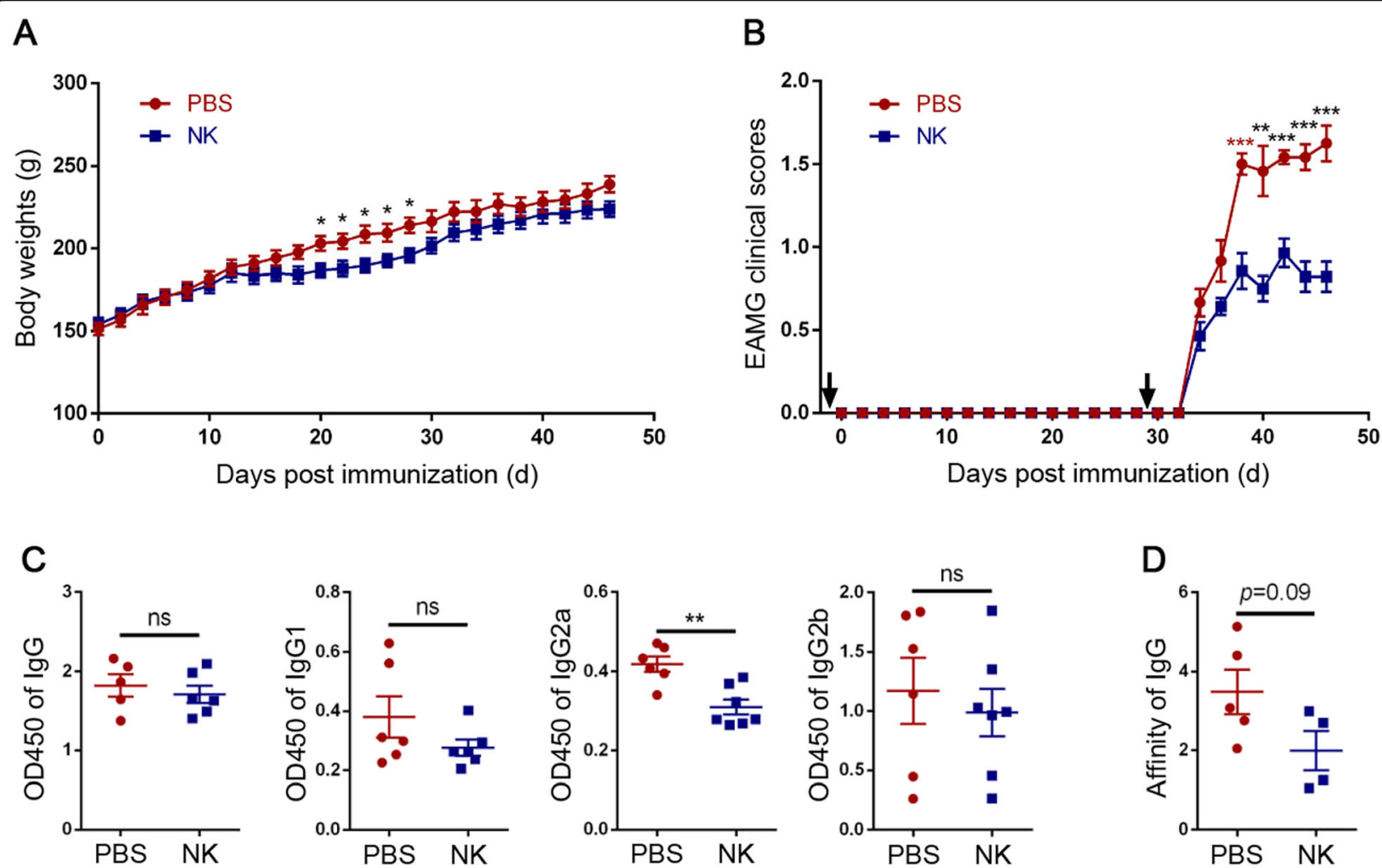

Fig 1 NK cell ameliorated EAMG symptoms and reduced serum anti-AChR97-116 lgG2a antibodies levels. NK cells were adoptively transferred into EAMG rats twice at the day before the first and second immunization, respectively. The body weights (a) and clinical scores (b) of NK celltreated rats $(n=7)$ and PBS-treated rats $(n=6)$ were recorded every other day after the first immunization. The rats were sacrificed at the 46th day post the first immunization, and the blood sera were collected. Serum anti-AChR97-116 lgG, IgG1, IgG2a, and IgG2b antibody levels were determined by ELISA (c). Anti-AChR97-116 lgG antibody affinity was as determined by the thiocyanate method (d). Data were presented as mean \pm SEM. Results were representatives of two independent experiments. Unpaired Student's $t$ test was used. Arrows mean intervention times. ${ }^{*} p<0.05,{ }^{*} p<0.01,{ }^{* * *} p<0.001$ 
groups (Fig. 1c). Interestingly, transient body weight loss from days 20 to 28 post-immunization (p.i.) was observed in NK cell-treated group (Fig. 1a).

\section{NK cell adoptive transfer reduces Th and germinal center $B$ cells in EAMG}

Considering that recent reports illustrated regulatory functions of NK cells in humoral immune response $[15,16]$, we decided to further examine the potential roles of NK cells in the regulation of Tfh cells and germinal center B cells. Consistently, EAMG rats treated with NK cells exhibited lower percentages of Tfh cells and germinal center B cells compared to untreated rats (Fig. 2a, b). A slight but statistically significant decrease of $B$ cell percentages in the NK cell-treated group was also observed (Fig. 2c, left). However, the percentages of memory $\mathrm{B}$ cells were not changed by NK cell treatment (Fig. 2c, right). The previous study illustrated Tfh cells with different cytokine profiles could modulate the affinity and isotype of the antibody response. Tfh1 cells, characterized by IFN- $\gamma$ production, were essential for IgG2a class switching [20]. Because adoptive transfer of NK cells led to decreased anti-AChR97-116 IgG2a antibody levels, we further determined whether NK cells could modulate Tfh cells subtypes, which include Tfh 1 and Tfh 17 cells. However, our results revealed that NK cell adoptive transfer regulated the percentage of neither Tfh1 cells nor Tfh17 cells (Fig. 2d, left). Also, the ratios of Tfh 1 to Tfh 17 were not different between the NK cell-treated and the control rats (Fig. 2d, right).

Dendritic cells (DCs) are the most potent professional antigen-presenting cells which play pivotal roles in driving Tfh cell differentiation [21-23]. To elucidate the mechanism of NK cells to regulate humoral immune response, we figure out whether NK cells could modulate the number or function of DCs. However, we did not observe either the number or phenotype changes of DCs between those two groups (Fig. 2e).

\section{NK cells inhibit $\mathrm{CD}^{+} \mathrm{T}$ cells and Tfh cells by increased apoptosis of $\mathrm{T}$ cells and Tfh cells in vitro}

Previous work demonstrated that NK cells could eliminate activated $\mathrm{CD}^{+}$and $\mathrm{CD} 8^{+} \mathrm{T}$ cells, which is mediated by TRAIL and perforin in a contact-dependent manner [11-13]. To address the effects of NK cells on Tfh cells, isolated NK cells were co-cultured with splenocytes for 3 days in vitro. Co-culturing rat splenic cells with NK cells led to a substantial reduction in the percentage of Tfh cells (Fig. 3a). The numbers of $\mathrm{CD} 4^{+} \mathrm{T}$ cells and Tfh cells were also decreased when mouse splenic cells were co-cultured with NK cells (Fig. 3b). However, the frequencies of mouse $\mathrm{Tfh}$ in $\mathrm{CD}_{4}^{+} \mathrm{T}$ cells were not changed (Fig. 3b). To investigate the mechanism of NK cells in regulating $\mathrm{T}$ cells and $\mathrm{Tfh}$ cells, the mouse splenocytes were stained with Annexin V after co-cultured with NK cells and found that NK cells increased apoptosis of $\mathrm{CD}^{+} \mathrm{T}$ cells (Fig. 3c).

To exclude the influence of other cell components in splenocytes which might mediate the suppressive effects of NK cells on Tfh cells, splenic $\mathrm{CD}^{+} \mathrm{T}$ cells were isolated and co-cultured with NK cells. Consistently, NK cells decreased Tfh cells (Fig. 3d) and induced the apoptosis of $\mathrm{CD}^{+} \mathrm{T}$ cells and Tfh cells (Fig. 3e). These results indicated NK cells directly inhibited $\mathrm{T}$ cells and Tfh cells by inducing their apoptosis.

\section{IL-15 increases NK cells and decreases CD4 ${ }^{+} \mathrm{T}$ cells and Tfh cells in vitro}

To further verify the inhibition effects of NK cells on Tfh cells, splenocytes were treated with IL-15, a wellknown NK cell stimulatory cytokine [24]. IL-15 could significantly increase NK cell percentages in the cultured splenocytes (Fig. 3f). Consistently, IL-15-treated groups had lower levels of $\mathrm{CD} 4^{+} \mathrm{T}$ cells and Tfh cells compared with control groups by inducing apoptosis of $\mathrm{T}$ cells (Fig. 3f, g), which confirmed from one perspective that NK cells could regulate Tfh cells. Additionally, IL-15 inhibited LPS-induced B cell proliferation (Fig. 3g), and this might attribute to its inhibiting effects on $\mathrm{CD} 4^{+} \mathrm{T}$ cells and Tfh cells.

\section{NK cells can be divided into $\mathrm{CXCR5}^{-}$and $\mathrm{CXCR}^{+} \mathrm{NK}$ subtypes}

CXCR5 molecular is expressed by B cells and Tfh cells, which directs those cells to localize to B cell follicles. Our results indicated NK cells could also be divided into CXCR5 $5^{-}$and $\mathrm{CXCR} 5^{+} \mathrm{NK}$ subtypes according to the expression of CXCR5 molecular. CXCR $5^{+} \mathrm{NK}$ cells were indeed shown in periphery blood, spleen, and lymph node and accounted for $0.63 \pm 0.03 \%, 2.44 \pm 0.17 \%$, and $9.75 \pm 0.96 \%$ of NK cells (means \pm SEM), respectively (Fig. 4a). CXCR5 ${ }^{+}$NK cells produced higher levels of IL17 than the CXCR5 subset, although comparable frequencies of IFN- $\gamma$-producing and CD25-positive cells were shown in both subsets (Fig. 4b). Furthermore, $\mathrm{CXCR}^{+} \mathrm{NK}$ cells exhibited higher expression level of ICOS (Fig. 4b) and lower of CD27 than their CXCR5 counterparts (Fig. 4c). Moreover, according to the expression level of CD27, both CXCR5 ${ }^{-}$and CXCR5 ${ }^{+} \mathrm{NK}$ cells could be divided into three subsets (CD27 $7^{\text {low }}$, $\mathrm{CD} 27^{\text {median }}$, and $\mathrm{CD} 27^{\text {hi }}$, respectively) (Fig. 4d). Indeed, $\mathrm{CXCR}^{+} \mathrm{NK}$ cells had higher frequencies of $\mathrm{CD} 27^{\text {median }}$ cells and lower frequencies of $\mathrm{CD} 27^{\text {hi }}$ cells compared with $\mathrm{CXCR}^{-} \mathrm{NK}$ cells, and these two NK cell subtypes had equal percentages of $\mathrm{CD} 27^{\text {low }}$ cells (Fig. 4d). Because CD27 is firstly expressed by immature NK cells and downregulated after maturation, these results indicated $\mathrm{CXCR}^{+}$NK cells might be more maturated than CXCR5 $5^{-}$NK cells. 


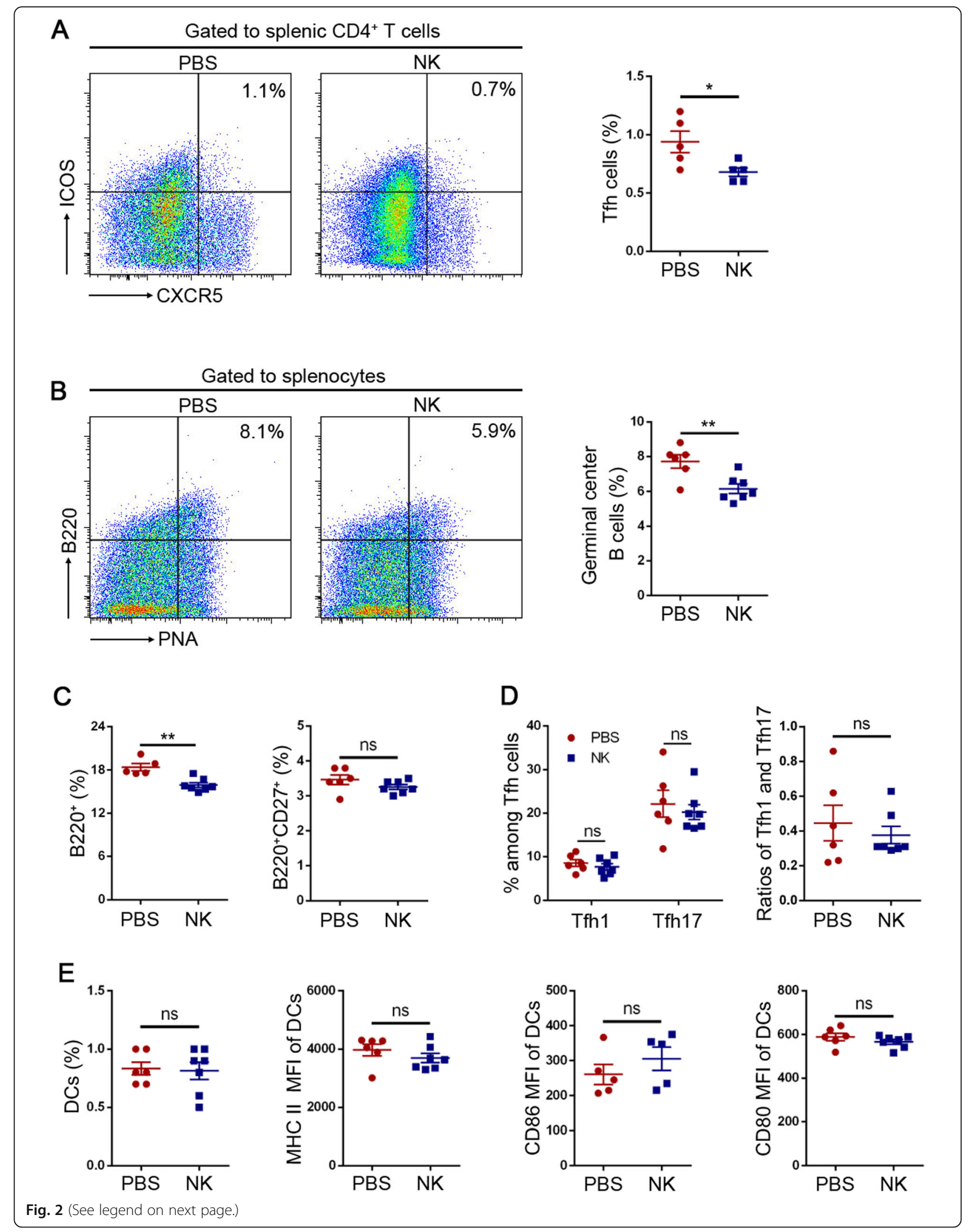


(See figure on previous page.)

Fig. 2 NK cell adoptive transfer reduced Tfh cells and germinal center B cells in EAMG. Rats were sacrificed at the 46th day post the first immunization, and the spleens were collected. The following results were representatives of two independent experiments. $\mathbf{a}$ Frequencies of Tfh cells $\left(\mathrm{CD}^{+}{ }^{+} \mathrm{CXCR5^{+ }} \mathrm{ICOS}^{+}\right)$among splenic $\mathrm{CD} 4^{+}$cells were downregulated by NK cell treatment. $\mathbf{b}$ Frequencies of germinal center $\mathrm{B}$ cells $\left(B 220^{+} \mathrm{PNA}^{+}\right)$among splenocytes were downregulated after NK cell adoptive transfer. $\mathbf{c}$ B cell $\left(B 220^{+}\right)$frequencies but not memory B cell $\left(B 220^{+} \mathrm{CD} 27^{+}\right)$frequencies among splenocytes were reduced after NK cell adoptive transfer. $\mathbf{d}$ NK cell adoptive transfer did not change IFN- $\gamma^{+}$ (Tfh1) or IL-17 (Tfh17) cell frequencies among Tfh cells $\left(\mathrm{CD}^{+} 4^{+} \mathrm{CXCR5^{+ }} \mathrm{ICOS}^{+}\right.$). e Frequencies and phenotypes of splenic DCs after NK cell treatment in EAMG. Dendritic cells were gated as CD103 ${ }^{+} \mathrm{MHC} \|^{+}$. The phenotypes of DCs referred to CD80, CD86, and MHC II expression levels, which were shown as MFI (mean fluorescent intensity). Data were presented as mean \pm SEM. Unpaired Student's $t$ test was used. ns means not significant, ${ }^{*} p<0.05,{ }^{* *} p<0.01$

\section{Distribution and migration of $\mathrm{CXCR5}^{-}$and $\mathrm{CXCR5}^{+} \mathrm{NK}$ cells}

To figure out the different functions of CXCR5 $5^{-}$and $\mathrm{CXCR}^{+} \mathrm{NK}$ cells in regulating humoral immune response, the distribution and migration of CXCR5 5 and $\mathrm{CXCR}^{+} \mathrm{NK}$ cells were investigated. To identify the localization of NK cells by immunofluorescence, CD161 and CXCR5 were stained with the corresponding antibodies. CD161 were used to indicate NK or natural killer $\mathrm{T}$ (NKT) cells. The molecular CXCR5 are mainly expressed by B cells; thus, it can be used as the marker for the B cell zone. Immunofluorescent results indicated $\mathrm{CD} 161^{+} \mathrm{CXCR}^{+}$cells were concentrated in the B cell zone while $\mathrm{CD} 161^{+} \mathrm{CXCR} 5^{-}$cells were mainly localized outside the B cell zone (Fig. 5a). This indicated that the $\mathrm{CXCR}^{+} \mathrm{NK}$ cells or NKT cells were localized in the B cell zone. Germinal centers are the sites in the B cell zone, which are formed after immune challenges and are essential for the antibody class switch and affinity maturation. To determine whether NK cells were localized in the geminal center, the frozen sections were stained for CD161 and germinal center with anti-CD161 antibody and PNA, respectively. Our results illustrated there were $\mathrm{CD} 161^{+}$cells in the lymph node PNA-positive area, suggesting the existence of NK cells or NKT cells in the germinal center (Fig. 5b). To directly assess whether NK cells could migrate into B cell zone, CFSE-labeled splenic NK cells were transferred to the immunized rats. The labeled NK cells were indeed found in the B cell zone of the spleen (Fig. 5c), which indicated some of NK cells could migrate into B cell zone. To figure out the difference of migration abilities between the CXCR5 and $\mathrm{CXCR}^{+} \mathrm{NK}$ cells, the CXCR5 ${ }^{-}$and $\mathrm{CXCR}^{+} \mathrm{NK}$ cells were sorted, labeled with CFSE, and transferred to different recipients. Results demonstrated that $\mathrm{CXCR} 5^{+}$ NK cells could more efficiently migrate into B cell zone than the CXCR5 subtype (Fig. 5d). A tiny number of NK cells were also observed in the B cell zone of spleen which received $\mathrm{CXCR} 5^{-} \mathrm{NK}$ cells. This might be attributed to the contaminated $\mathrm{CXCR} 5^{+} \mathrm{NK}$ cells among the purified $\mathrm{CXCR}^{-} \mathrm{NK}$ cells. These results indicated $\mathrm{CXCR}^{-}$and $\mathrm{CXCR}^{+} \mathrm{NK}$ cells might regulate immune response in different sites of the secondary lymphoid organs. CXCR5 ${ }^{-} \mathrm{NK}$ cells might regulate immune response outside the B cell zone, while $\mathrm{CXCR} 5^{+} \mathrm{NK}$ cells might migrate into $\mathrm{B}$ cell follicles and regulate humoral immune response in the $\mathrm{B}$ cell area.

\section{Tfh cells and germinal center cells are positively correlated with CXCR5 ${ }^{+}$NK cells}

To investigate the potential impact of $\mathrm{CXCR}^{-}$and $\mathrm{CXCR}^{+} \mathrm{NK}$ cells on Tfh cells, we firstly evaluated the correlation between $\mathrm{Tfh}$ cells and $\mathrm{CXCR}^{+} \mathrm{NK}$ cells in rat EAMG models by Spearman correlation test. Interestingly, Tfh cell frequencies had a positive correlation with $\mathrm{CXCR}^{+} \mathrm{NK}$ cell frequencies in EAMG rats (Fig. 6a, left). Consistently, Tfh cell frequencies were negatively correlated with the ratios of $\mathrm{CXCR}^{-}$to $\mathrm{CXCR} 5^{+} \mathrm{NK}$ cells (Fig. 6a, right). Similar results were also observed in experimental autoimmune neuritis (EAN) model rats (Fig. 6b). In addition, the frequencies of germinal center $\mathrm{B}$ cells were also positively correlated with $\mathrm{CXCR} 5^{+} \mathrm{NK}$ cell frequencies (Fig. 6c, left) and negatively correlated with the ratios of CXCR5 5 to $\mathrm{CXCR}^{+}$NK cells in EAMG rats (Fig. 6c, right). These results indicated that $\mathrm{CXCR} 5^{-}$and $\mathrm{CXCR} 5^{+} \mathrm{NK}$ cells might have different roles in regulating Tfh cells or germinal center B cells.

\section{CXCR5 ${ }^{-}$NK cells but not CXCR5 ${ }^{+}$NK cells suppressed Tfh cells}

To directly address the different roles of CXCR5 ${ }^{-}$and $\mathrm{CXCR}^{+}$NK cells on Tfh cells, mouse splenic CXCR5 ${ }^{+}$ NK or CXCR5 ${ }^{-}$NK cells were co-cultured with splenocytes and found that CXCR5 $5^{-} \mathrm{NK}$ cells but not CXCR5 ${ }^{+}$ NK and could reduce CD4 ${ }^{+} \mathrm{T}$ cell and Tfh cell numbers (Fig. 6d, e). Similar results were obtained when isolated splenic $\mathrm{CD}^{+} \mathrm{T}$ cells were co-cultured with splenic $\mathrm{CXCR}^{-}$or $\mathrm{CXCR}^{+} \mathrm{NK}$ cells (Fig. 6f). Further analysis illustrated that compared with $\mathrm{CXCR}^{-} \mathrm{NK}$ cells, $\mathrm{CXCR}^{+} \mathrm{NK}$ cells increased $\mathrm{ICOS}^{+}$cells percentages among $\mathrm{CD}^{+} \mathrm{CXCR}^{+} \mathrm{T}$ cells (Fig. 6g). Meanwhile, $\mathrm{CXCR}^{+}$but not CXCR5 $5^{-} \mathrm{NK}$ cells upregulated ICOS mean fluorescent intensity (MFI) of Tfh cells (Fig. 6h). These results illustrated that $\mathrm{CXCR}^{-} \mathrm{NK}$ cells and $\mathrm{CXCR}^{+} \mathrm{NK}$ cells have different functions in regulate immune response and CXCR5 $5^{-} \mathrm{NK}$ cells could more efficiently suppressed Tfh cells than CXCR $5^{+} \mathrm{NK}$ cells. 


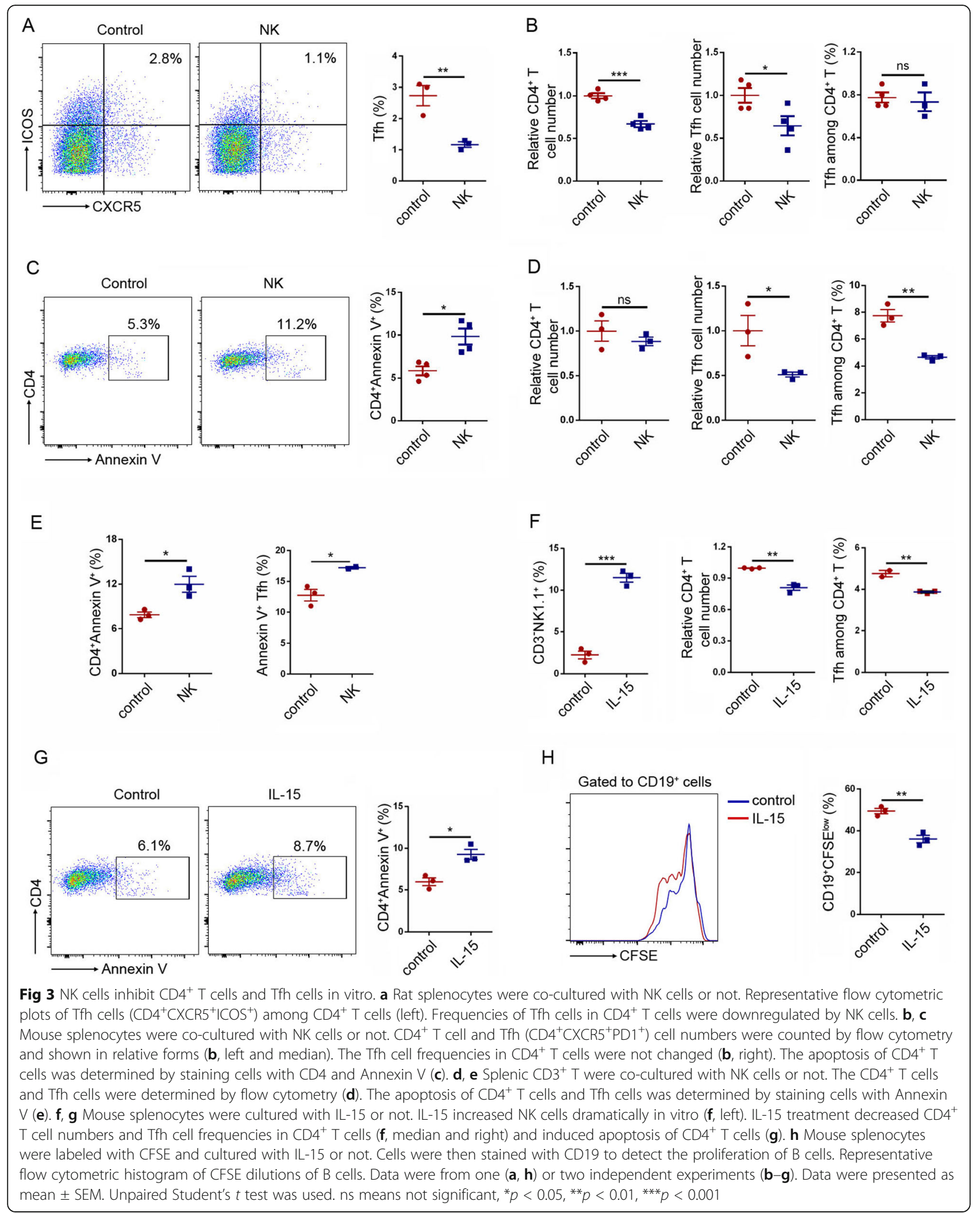



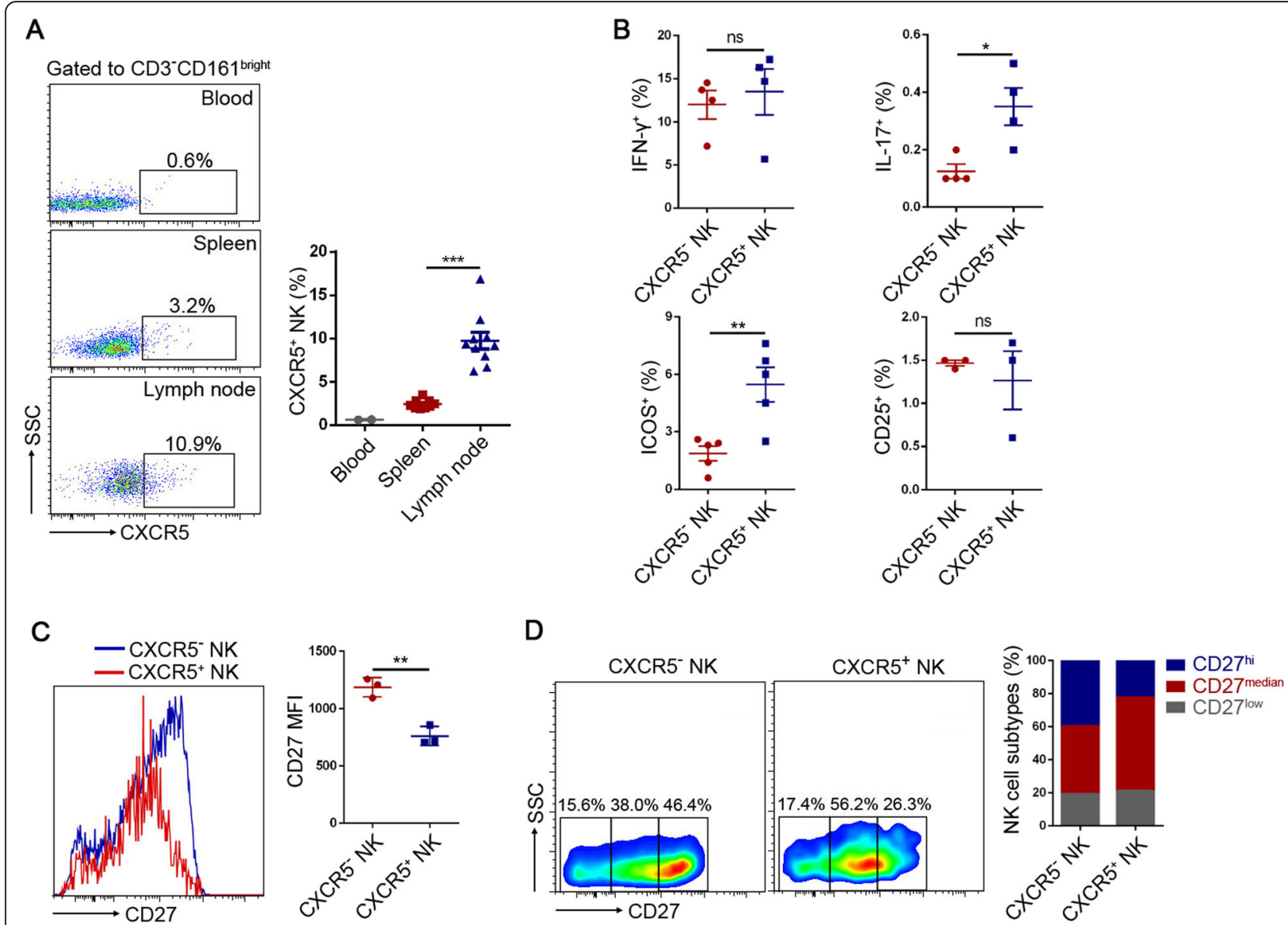

Fig. 4 Phenotype differences between CXCR5 and CXCR5 ${ }^{+}$NK cells. a Frequencies of $C X C R 5^{+}$cell among blood, lymph node, and spleen $\mathrm{CD}^{-} \mathrm{CD} 161^{\text {bright }} \mathrm{NK}$ cells. Data were presented as mean \pm SEM. One-way ANOVA. ${ }^{* *} p<0.01$. b Frequencies of IFN- $\gamma^{+}, \mathrm{IL}^{*} 17^{+}, \mathrm{ICOS}^{+}$, and $_{\mathrm{CD} 25^{+}}$ cells within rat CXCR5 ${ }^{-}$and CXCR5 $^{+}$NK cells. Data were presented as mean \pm SEM. Results were representatives of two or three independent experiments. Unpaired Student's $t$ test was used. ns means not significant, ${ }^{*} p<0.05$, ${ }^{*} p<0.01$. c Representative flow cytometric histogram of the expression of CD27 on rat CXCR5 and CXCR5 ${ }^{+}$NK cells (left). Mean fluorescent intensity (MFI) of CD27 expression of rat CXCR5 and CXCR5 ${ }^{+}$ NK cells (right). Data were presented as mean \pm SEM. Three independent experiments. Unpaired Student's $t$ test was used. **p $<0.01$. d Divide rat CXCR5 ${ }^{-}$and $\mathrm{CXCR5}^{+}$NK cells into three subsets $\left(\mathrm{CD} 27^{\text {low }}, \mathrm{CD} 27^{\text {median }}\right.$, and CD27 $7^{\text {hi }}$ ) according to CD27 expression levels (left). Frequencies of $\mathrm{CD} 27^{\text {low }}, \mathrm{CD}_{2} 7^{\text {median }}$, and $\mathrm{CD} 27^{\text {hi }}$ cells among CXCR5 and CXCR5 ${ }^{+}$NK cells (right). Three independent experiments. Data were presented as mean

\section{Discussion}

Growing evidence demonstrates that cellular immunology such as $\mathrm{CD}^{+}$and $\mathrm{CD} 8^{+} \mathrm{T}$ cells plays critical roles in the pathogenesis of MG [25]. However, the regulatory activity of NK cells, the innate immune component of cellular immune system, has been given less experimental attention. NK cells play important roles in the pathogenesis of several autoimmune diseases by modulating other components of the immune system. In this study, we investigated the roles and mechanism of NK cells in regulating EAMG by adoptive transferring splenic NK cells to recipient rats and by co-culturing NK cells or their subtypes with splenocytes or $\mathrm{T}$ cells in vitro.

NK cell treatment ameliorated EAMG symptoms, and this was associated with a reduction of anti-AChR97116 IgG2a antibody levels and germinal center B cells. Previous study showed that depleting NK cell with anti-
NK1.1 antibody decrease onset and severity of murine EAMG, which is contrary to our observations [26]. This discrepancy might attribute to the difference in interventions and animal models. NK1.1 are not exclusively expressed by NK cells, but also by some myeloid cells and $\mathrm{T}$ cells [27]. Thus, administration of anti-NK1.1 antibody might change these NK1.1-positive components, which make the results complicated to analyze.

Tfh cells are highly specified $\mathrm{CD}^{+}{ }^{+} \mathrm{T}$ cell subset responsible for $\mathrm{B}$ cell proliferation, differentiation, and antibody production. In line with the decreased serum antibody levels and germinal center B cell percentages in NK-treated rats, our study indicated NK cell adoptive transfer reduced the percentages of Tfh cells. Further experiments confirmed these observations, as NK cells directly suppressed $\mathrm{T}$ cells and $\mathrm{Tfh}$ cells in vitro. Mechanically, NK cells could induce the apoptosis $\mathrm{T}$ 

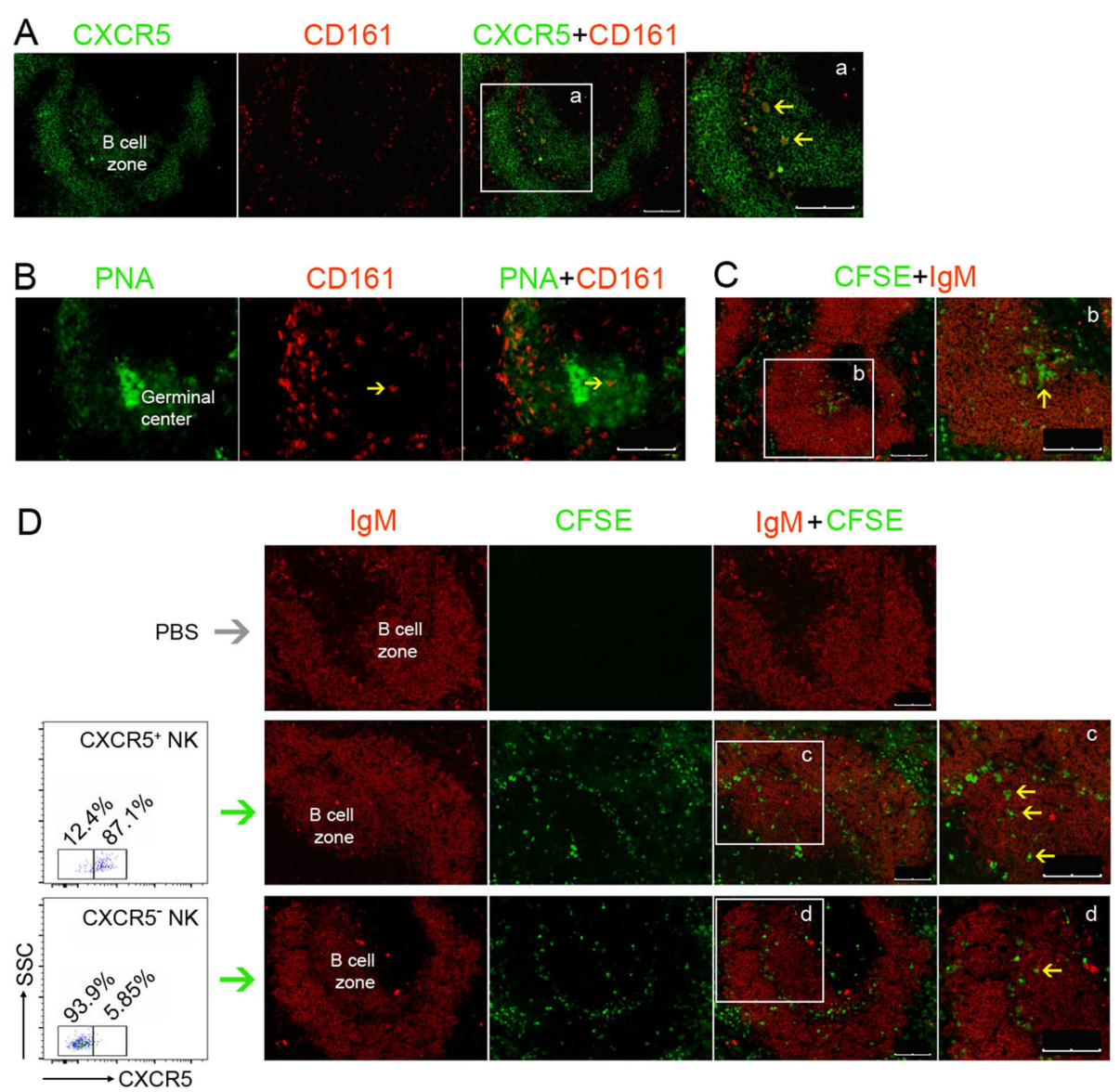

Fig. 5 Distribution and migration of $C X C R 5^{+}$and $C X C R 5^{-}$NK cells. a Fluorescence image of a spleen from EAMG rat. Red indicated CD161, and green indicated CXCR5 (B cell zone). Arrowhead indicated CD $161^{+}$CXCR5 ${ }^{+}$cells in the B cell zone. Scale bar, $100 \mu m$. b Fluorescence image of EAMG rat lymph node. Red indicated CD161, and green (PNA) indicated germinal center area. Arrowhead indicated CD $161^{+}$cells in the germinal center area. Scale bar, 100 um. c Some of NK cells migrated into B cell zone after adoptive transfer. NK cells, sorted by MACS sorting, were labeled with CFSE (green) and transferred into EAMG rats. Two days later, the spleen was harvested and stained with anti-lgM antibody (red). Arrowhead indicated NK cells in B cell zone, scale bar, $100 \mu \mathrm{m}$. d FACS sorted CXCR5 ${ }^{+}$and CXCR5 $5^{-}$NK cells were labeled with CFSE (green) and transferred into EAMG rats. Two days later, the spleen was harvested and stained with the anti-lgM antibody (red). Arrowhead indicated NK cells in B cell zone, scale bar, $100 \mu \mathrm{m}$

cells and Tfh cells. Indeed, antigen-activated T lymphocytes express cell-surface NKG2D ligands and become susceptible to autologous NK cell lysis [28]. NK cells also kill the $\mathrm{CD} 4^{+} \mathrm{T}$ cell response through a TRAILdependent mechanism [11]. Previous studies demonstrated NK cells could also induce the apoptosis of neutrophil and eosinophil [29, 30]. Thus, cytotoxicity against other immune cells may serve as a general mechanism by which NK cells limit the inflammation and autoimmune response.

However, the usage of NK cell adoptive transfer for treating MG may be confined by the limited number of autologous NK cells which could be obtained. Although various culture methods have been developed to obtain sufficient number NK cell, ex vivo treatment may modulate the immunophenotypes and the subsequent functions of NK cells. A feasible alternative method is to activate or expand NK cells in vivo. IL-15 and its genetically modified super-agonist ALT-803, which has been used in clinical trials for treating tumors, can activate and expand NK cells and may be a promising candidate for treating MG. Indeed, IL-15 inhibited $\mathrm{CD} 4^{+} \mathrm{T}$ cells and Tfh cells in vitro. Similarly, IL-15 could increase apoptosis of $\mathrm{CD}^{+} \mathrm{T}$ cells. Additionally, IL-15 also inhibited LPS-induced B cell proliferation, and this might attribute to its inhibiting effects on $\mathrm{T}$ and $\mathrm{Tfh}$ cells. This was consistent with a previous study that NK cell could suppress the proliferation of B cells [31]. Thus, NK cells and their activators might be the new therapeutics methods in treating MG and other antibody-mediated autoimmune diseases in the future.

NK cells are the heterogenous lymphocytes with different phenotypes and functions. Different NK cell subtypes seems to play different even opposite roles in 


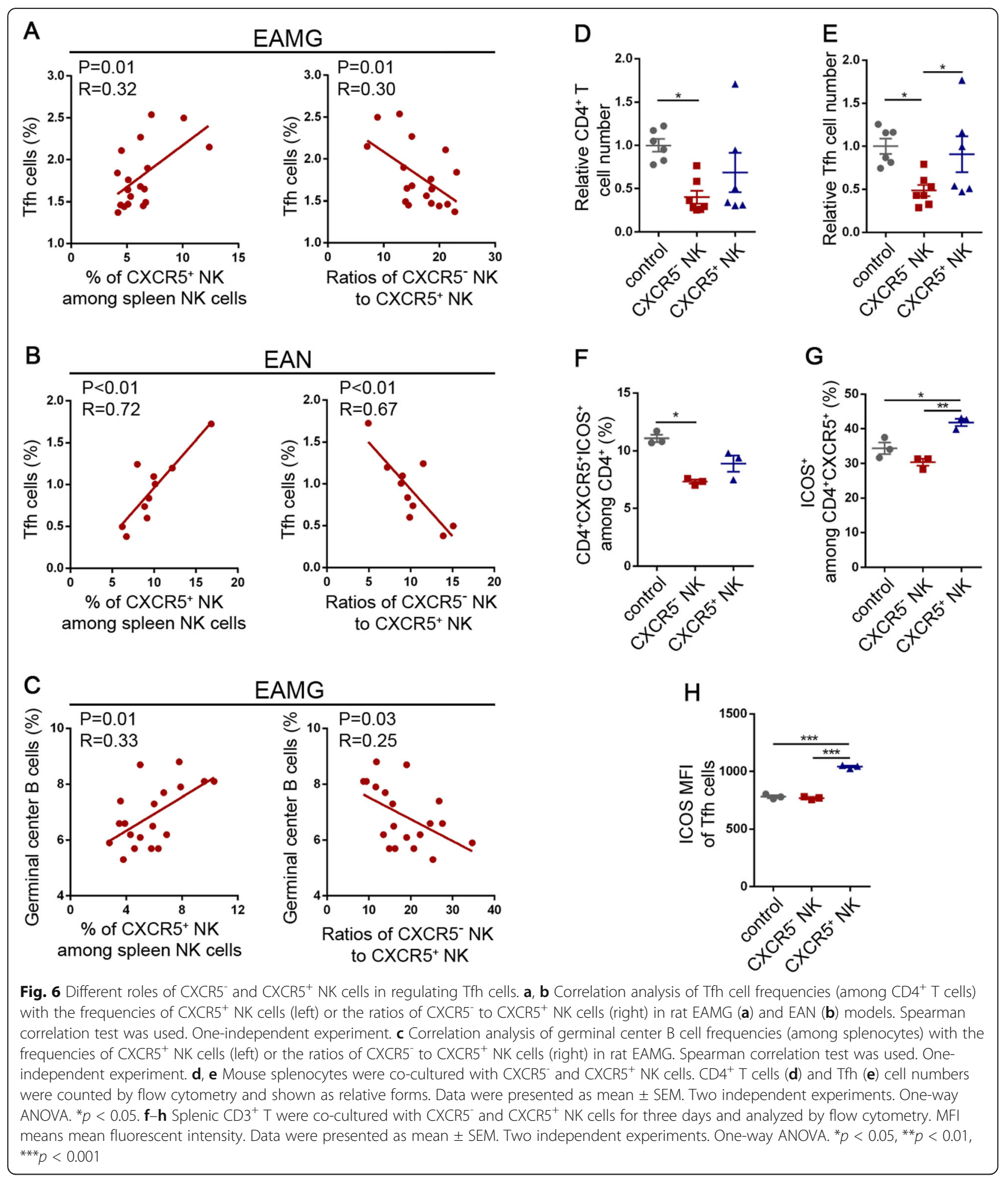

regulating immune response [32]. Our results indicated, according to the expression of CXCR5 molecular, NK cells could be divided into CXCR $^{-}$and CXCR $^{+}$NK subtypes. Phenotype analysis illustrated that, compared with $\mathrm{CXCR}^{-}$subset, CXCR5 ${ }^{+} \mathrm{NK}$ cells exhibited higher levels of ICOS and lower levels of CD27 and had a higher frequency of IL-17-producing cells. As CXCR5 is the chemokine receptor of CXCL13, which guides Tfh and $\mathrm{B}$ cells to migrate to $\mathrm{B}$ cell follicles, it is reasonable to assume that $\mathrm{CXCR}^{+} \mathrm{NK}$ cells could migrate to B cell 
area to modulate humoral response. One pioneer study showed that $\mathrm{CXCR} 5^{+} \mathrm{NK}$ could enter in lymph node follicles, and exert efficient control of viral replication within lymph nodes [33]. Our study indicated CXCR5 ${ }^{+}$ NK cells could more efficiently migrate into B cell zone than the CXCR5 5 subtype. More importantly, the frequencies of Tfh cells and germinal center B cells were positively correlated with $\mathrm{CXCR} 5^{+} \mathrm{NK}$ cell frequencies in EAMG rats. In addition, the frequencies of Tfh cells and germinal center $\mathrm{B}$ cells were negatively correlated with the ratios of $\mathrm{CXCR}^{-}$to $\mathrm{CXCR}^{+} \mathrm{NK}$ cells. These results indicated that $\mathrm{CXCR}^{-}$and $\mathrm{CXCR}^{+} \mathrm{NK}$ cells might have different roles in regulating Tfh cells or germinal center B cells. Ex vivo studies showed that CXCR5 ${ }^{-}$NK cells downregulated Tfh cells percentages more efficiently than $\mathrm{CXCR}^{+} \mathrm{NK}$ cells. Compared with CXCR5 ${ }^{-}$NK cells, CXCR5 ${ }^{+}$NK cells could enhance ICOS expression on Tfh cells. As ICOS is critical for antibody class switching [34], CXCR $5^{+} \mathrm{NK}$ cells might exhibit promotive effects on humoral immune response. As a whole, different NK cell subtypes exhibit different effects on humoral immune response and CXCR5 NK cells but not CXCR5 ${ }^{+}$NK cells suppressed Tfh cells and inhibited the autoimmune response in EAMG models.

\section{Conclusions}

Our results indicated NK cells ameliorated EAMG symptoms by suppressing Tfh cells and germinal center $\mathrm{B}$ cells, and this might attribute to the inhibitory role of CXCR5 ${ }^{-}$NK cells but not $\mathrm{CXCR}^{+}$NK cells. The function of $\mathrm{CXCR}^{+} \mathrm{NK}$ cells on Tfh and $\mathrm{B}$ cells need to be investigated in detail in the future, as the environments of ex vivo studies could not stringently simulate the micro-environments in vivo.

\footnotetext{
Abbreviations

AChR: Acetylcholine receptor; BPM: Bovine peripheral myelin; CFSE: Carboxyfluorescein diacetate, succinimidyl ester; CXCR5: C-X-C chemokine receptor 5; DCs: Dendritic cells; EAE: Experimental autoimmune encephalomyelitis; EAMG: Experimental autoimmune myasthenia gravis; EAN: Experimental autoimmune neuritis; ICOS: Inducible co-stimulator; LPS: Lipopolysaccharide; MFI: Mean fluorescent intensity; MG: Myasthenia gravis; NK cells: Natural killer cells; PBS: Phosphate buffer saline; PD1: Programmed death 1; PNA: Peanut agglutinin; Tfh cells: Follicular helper T cells; TRAIL: TNF-related apoptosis-inducing ligand
}

\section{Acknowledgements}

Not applicable

\section{Authors' contributions}

RSD designed, analyzed, and provided overall guidance for the experiments. $C L Y$ designed, performed, and analyzed the experiments and wrote the manuscript. PZ, RTL, NZ, MZ, HL, and TD performed the experiments. XLL and YCD analyzed the experiments. All authors contributed to the experiments and read and approved the final manuscript.

\section{Funding}

This work was supported by grants from the National Natural Science Foundation of China (81471222) and Taishan Scholars Construction Engineering of Shandong Province (ts20130914).

\section{Availability of data and materials}

The datasets used and/or analyzed during the current study are available from the corresponding author on reasonable request.

\section{Ethics approval and consent to participate}

All the animal experimental protocols were approved by the Ethics Committee of Shandong Provincial Qianfoshan Hospital.

\section{Consent for publication}

Not applicable

\section{Competing interests}

The authors declare that they have no competing interests.

\section{Author details}

'Department of Neurology, Shandong Provincial Qianfoshan Hospital, Shandong University, No. 16766, Jingshi Road, Jinan 250014, People's Republic of China. ${ }^{2}$ Department of Neurology, The First Affiliated Hospital of Shandong First Medical University, Jinan 250014, People's Republic of China. ${ }^{3}$ College of Basic Medical Sciences, Shandong University of Traditional Chinese Medicine, Jinan 250355, People's Republic of China.

Received: 8 September 2019 Accepted: 20 December 2019

Published online: 29 December 2019

\section{References}

1. Romi F, Hong Y, Gilhus NE. Pathophysiology and immunological profile of myasthenia gravis and its subgroups. Curr Opin Immunol. 2017;49:9-13.

2. Gilhus NE. Myasthenia gravis. N Engl J Med. 2017;376:e25.

3. Ueno H. T follicular helper cells in human autoimmunity. Curr Opin Immunol. 2016;43:24-31.

4. Luo C, Li Y, Liu W, Feng H, Wang H, Huang $X$, et al. Expansion of circulating counterparts of follicular helper T cells in patients with myasthenia gravis. J Neuroimmunol. 2013;256:55-61.

5. Zhang CJ, Gong Y, Zhu W, Qi Y, Yang CS, Fu Y, et al. Augmentation of circulating follicular helper T cells and their impact on autoreactive B cells in Myasthenia gravis. J Immunol. 2016:197:2610-7.

6. Zhang X, Liu S, Chang T, Xu J, Zhang C, Tian F, et al. Intrathymic Tfh/B cells interaction leads to ectopic GCs formation and anti-AChR antibody production: Central Role in Triggering MG Occurrence. Mol Neurobiol. 2016; 53:120-31.

7. Xin N, Fu L, Shao Z, Guo M, Zhang X, Zhang Y, et al. RNA interference targeting $\mathrm{BCl}-6$ ameliorates experimental autoimmune myasthenia gravis in mice. Mol Cell Neurosci. 2014:58:85-94.

8. Freud AG, Mundy-Bosse BL, Yu J, Caligiuri MA. The broad spectrum of human natural killer cell diversity. Immunity. 2017:47:820-33.

9. Crome SQ, Lang PA, Lang KS, Ohashi PS. Natural killer cells regulate diverse T cell responses. Trends Immunol. 2013:34:342-9.

10. Lunemann A, Lunemann JD, Munz C. Regulatory NK-cell functions in inflammation and autoimmunity. Mol Med. 2009;15:352-8.

11. Schuster IS, Wikstrom ME, Brizard G, Coudert JD, Estcourt MJ, Manzur M, et al. TRAIL+ NK cells control CD4+ T cell responses during chronic viral infection to limit autoimmunity. Immunity. 2014;41:646-56.

12. Lang PA, Lang $\mathrm{KS}, \mathrm{Xu} \mathrm{HC}$, Grusdat M, Parish IA, Recher M, et al. Natural killer cell activation enhances immune pathology and promotes chronic infection by limiting CD8+ T-cell immunity. Proc Natl Acad Sci U S A. 2012;109: $1210-5$.

13. Waggoner SN, Taniguchi RT, Mathew PA, Kumar V, Welsh RM. Absence of mouse 2B4 promotes NK cell-mediated killing of activated CD8+ T cells, leading to prolonged viral persistence and altered pathogenesis. J Clin Invest. 2010;120:1925-38.

14. Jiang W, Li D, Han R, Zhang C, Jin WN, Wood K, et al. Acetylcholineproducing NK cells attenuate CNS inflammation via modulation of infiltrating monocytes/macrophages. Proc Natl Acad Sci U S A. 2017;114: E6202-11.

15. Cook KD, Kline HC, Whitmire JK. NK cells inhibit humoral immunity by reducing the abundance of $\mathrm{CD} 4+\mathrm{T}$ follicular helper cells during a chronic virus infection. J Leukoc Biol. 2015:98:153-62.

16. Rydyznski CE, Cranert SA, Zhou JQ, Xu H, Kleinstein SH, Singh H, et al. Affinity maturation is impaired by natural killer cell suppression of germinal centers. Cell Reports. 2018;24:3367-73 e3364. 
17. Zhang P, Yang CL, Liu RT, Li H, Zhang M, Zhang N, et al. Toll-like receptor 9 antagonist suppresses humoral immunity in experimental autoimmune myasthenia gravis. Mol Immunol. 2018;94:200-8.

18. Wang SX, Yang CL, Zhang M, Zhang P, Liu RT, Zhang N, et al. Sulfatides ameliorate experimental autoimmune neuritis by suppressing Th1/Th17 cells. J Neuroimmunol. 2019;326:55-61.

19. Li H, Wang CC, Zhang M, Li XL, Zhang P, Yue LT, et al. Statin-modified dendritic cells regulate humoral immunity in experimental autoimmune myasthenia gravis. Mol Cell Neurosci. 2015;68:284-92.

20. Reinhardt RL, Liang HE, Locksley RM. Cytokine-secreting follicular T cells shape the antibody repertoire. Nat Immunol. 2009:10:385-93.

21. Yao C, Zurawski SM, Jarrett ES, Chicoine B, Crabtree J, Peterson EJ, et al. Skin dendritic cells induce follicular helper T cells and protective humoral immune responses. J Allergy Clin Immunol. 2015;136:1387-97 e1381-1387.

22. Kim SJ, Schatzle S, Ahmed SS, Haap W, Jang SH, Gregersen PK, et al. Increased cathepsin S in Prdm1(-/-) dendritic cells alters the TFH cell repertoire and contributes to lupus. Nat Immunol. 2017;18:1016-24.

23. Levin C, Bonduelle O, Nuttens C, Primard C, Verrier B, Boissonnas A, et al. Critical role for skin-derived migratory DCs and Langerhans cells in TFH and GC responses after intradermal immunization. J Invest Dermatol. 2017;137: 1905-13.

24. Freund-Brown J, Chirino L, Kambayashi T. Strategies to enhance NK cell function for the treatment of tumors and infections. Crit Rev Immunol. 2018:38:105-30.

25. Zhang GX, Xiao BG, Bakhiet $M$, van der Meide $P$, Wigzell $H$, Link $H$, et al. Both CD4+ and CD8+ T cells are essential to induce experimental autoimmune myasthenia gravis. J Exp Med. 1996;184:349-56.

26. Shi FD, Wang HB, Li H, Hong S, Taniguchi M, Link H, et al. Natural killer cells determine the outcome of B cell-mediated autoimmunity. Nat Immunol. 2000;1:245-51.

27. Wikenheiser DJ, Brown SL, Lee J, Stumhofer JS. NK1.1 Expression defines a population of CD4(+) effector $T$ cells displaying Th1 and Tfh cell properties that support early antibody production during Plasmodium yoelii infection. Front Immunol. 2018;9:2277.

28. Cerboni C, Zingoni A, Cippitelli M, Piccoli M, Frati L, Santoni A. Antigenactivated human T lymphocytes express cell-surface NKG2D ligands via an ATM/ATR-dependent mechanism and become susceptible to autologous NK- cell lysis. Blood. 2007;110:606-15.

29. Kim JH, Choi GE, Lee BJ, Kwon SW, Lee SH, Kim HS, et al. Natural killer cells regulate eosinophilic inflammation in chronic rhinosinusitis. Sci Rep. 2016;6: 27615.

30. Thoren FB, Riise RE, Ousback J, Della Chiesa M, Alsterholm M, Marcenaro E, et al. Human NK Cells induce neutrophil apoptosis via an NKp46- and Fasdependent mechanism. J Immunol. 2012;188:1668-74.

31. Commes $T$, Clofent $G$, Jourdan M, Bataille R, Klein B. Human natural killer cells suppress the proliferation of B cells. Immunol Lett. 1990;24:57-61.

32. Tian Z, Gershwin ME, Zhang C. Regulatory NK cells in autoimmune disease. J Autoimmun. 2012;39:206-15.

33. Huot N, Jacquelin B, Garcia-Tellez T, Rascle P, Ploquin MJ, Madec Y, et al. Natural killer cells migrate into and control simian immunodeficiency virus replication in lymph node follicles in African green monkeys. Nat Med. 2017:23:1277-86.

34. McAdam AJ, Greenwald RJ, Levin MA, Chernova T, Malenkovich N, Ling V, et al. ICOS is critical for CD40-mediated antibody class switching. Nature. 2001:409:102-5.

\section{Publisher's Note}

Springer Nature remains neutral with regard to jurisdictional claims in published maps and institutional affiliations.

Ready to submit your research? Choose BMC and benefit from:

- fast, convenient online submission

- thorough peer review by experienced researchers in your field

- rapid publication on acceptance

- support for research data, including large and complex data types

- gold Open Access which fosters wider collaboration and increased citations

- maximum visibility for your research: over $100 \mathrm{M}$ website views per year

At $\mathrm{BMC}$, research is always in progress.

Learn more biomedcentral.com/submissions 